\title{
Development of a Small-Scale Plastic Recycling Technology and a Special Filament Product for 3D Printing
}

\author{
A. OUSSAI ${ }^{1}$, Z. BÁRTFAI ${ }^{1}$, L. KÁtAI ${ }^{2}$, I. SZALKAI \\ 1Szent István University, Faculty of Mechanical Engineering \\ Instutute of Mechanics and Machinery \\ 2FKF Nonprofit ZRT \\ E-mail: alaoussai@gmail.com
}

Keywords: Recycling, plastic, polymers, 3D printing, filaments

Abstract. In our days, the fight against pollution has become a real challenge for the state. recycling is one of the solutions that is adopted in several nations to reduce the rate of plastic discarded in nature.

The amount of plastic waste has been increasing for decades contributing to the environmental pollution that is one of the most serious problem of the mankind. according to the statistics not only the household plastic waste, but the industry discharge is increasing because the utilization of plastic as a raw material is more and more extending. plastic can be found in a lot of products, huge number of bottles, plastic bags, computers, auto parts are sold every day.

The current applications for using recycled plastics in fabrication and design are fairly limited, on a small scale, plastics (such as abs, HDPe1, or Pe2t) are shredded and formed into pellets, and then either extruded into lament to be used in existing $3 d$ printers, or injection molded into small parts and pieces of larger components. at a large scale, recycled HDPE is melted into sheets and either used directly as sheets in construction, or then heat formed from a sheet into components for construction. these methods of fabrication using recycled plastics are the norm because of their straightforward processes. nevertheless, each method leaves some complexity to be desired.

This paper we study the types of plastics and diagnose the pollution caused by the latter. this allowed us to design and size a recycling station of plastic into filaments for three-dimensional printers. this station which will contribute to the fight against pollution. the station consists of two machines for grinding of the plastic and the other for the extrusion of the desired filaments. we were able to make a theoretical academic study on both machines and also we designed with solidworks 2015. The theoretical study is spread of the mechanical calculations necessary to the design and validation of the structure using the tools. as the prospect of this project, we want to complete the achievement of this station while completing the crusher and extruder mechanically. then switch to electric and electronic parts (introduction of engines, sensors and wiring...).

In the case of waste plastics that are recyclable and reusable. the most widely used are polyethylene terephthalate (pet, used for synthetic fibers and water bottles), and second high-density polyethylene (hdpe, used for jugs, bottle caps, water pipes).

\footnotetext{
${ }^{1}$ second high-density polyethylene

${ }^{2}$ polyethylene terephthalate
} 


\section{Introduction}

Having studied the applied plastic recycling technologies, materials for 3D printing and the 3D printing technologies It realized that the quantity and quality of plastic waste differs from country to country and from company to other. ${ }^{1}$

In the case of waste plastics that are recyclable and reusable. The most widely used are polyethylene terephthalate (PET, used for synthetic fibers and water bottles), and second high-density polyethylene (HDPE, used for jugs, bottle caps, water pipes).

The current applications for using recycled plastics in fabrication and design are fairly limited, on a small scale, plastics that are the plastics (such as ABS, HDPE, or PET) are shredded and formed into pellets, and then either extruded into lament to be used in existing 3D printers, or injection molded into small parts and pieces of larger components. At a large scale, recycled HDPE is melted into sheets and either used directly as sheets in construction, or then heat formed from a sheet into components for construction. These methods of fabrication using recycled plastics are the norm because of their straightforward processes. Nevertheless, each method leaves some complexity to be desired (1) .

Regarding the recycling technology, the previous methods of recycling and recovery routes for solid plastic waste are detailed and discussed covering:

- re-extrusion

- mechanical treatment

- chemical treatment

- energy recovery

Based on the previous research I performed on this field, the main objective of my project is the contribution to the development of sustainable, effective technologies on the field of plastic recycling, both from the economic and ecological point of view.

Table 1. Summary of mechanical recycling studies ${ }^{2}$

\begin{tabular}{|c|c|c|}
\hline Reference & Main single-polymer plastics used & Comments \\
\hline Kowalska et al. (2002) & $\begin{array}{l}\text { PP (Malen P F-401) } \\
\text { Waste LDPE } \\
\text { Waste PVC } \\
\text { Reclaimed LDPE films } \\
\text { Suspension PVC }\end{array}$ & $\begin{array}{l}\text { - Thermoplastics were mixed and extruded with fillers (waste rubber granulate, whiting, cel- } \\
\text { lulose fibres and wood flour) to obtain an optimum blend composition } \\
\text { - The blend contained }<50 \text { wt\% of secondary LDPE, }>50 \text { wt\% of comminute rubber scrap and } \\
<0.1 \text { wt\% of blowing agent } \\
\text { - } 3-4 \mathrm{l} / \mathrm{h} \text { of water permutation was achieved with the blend making it a satisfactory mixture }\end{array}$ \\
\hline Strapasson et al. (2005) & $\begin{array}{l}\text { PP/LDPE blends }(0 / 100,25 / 75,50 / 50,75 / 25 \text { and } \\
100 / 0 \mathrm{wt} / \mathrm{wt} \%) \text { via injection moulding }\end{array}$ & $\begin{array}{l}\text { - Thermoplastics were mixed and extruded with fillers (waste rubber granulate, whiting, cel- } \\
\text { louse fibres and wood flour) to obtain an optimum blend composition }\end{array}$ \\
\hline Lei et al. (2007) & RHDPE & $\begin{array}{l}\text { - Composites based on RHDPE and natural fibres, made through melt blending and compres- } \\
\text { sion moulding were studied, so were the effects of fibres and coupling agent (type/concen- } \\
\text { tration) on the composite properties } \\
\text { - The use of MAPE, CAPE and TDM improved the compatibility between the fibre and RHDPE, } \\
\text { and mechanical properties of the resultant composites compared well with those of virgin } \\
\text { HDPE composites }\end{array}$ \\
\hline Meran et al. (2008) & LDPE, HDPE and PP & $\begin{array}{l}\text { - The tensile strength relation was monitored in PP since the loss of mechanical and physical } \\
\text { properties did not exceed } 50 \% \text { in the films studied }\end{array}$ \\
\hline Brachet et al. (2008) & PP & $\begin{array}{l}\text { - Modification of mechanical properties of recycled PP from post-consumer containers with } \\
\text { the addition of stabilizers, elastomer (EOR) and } \mathrm{CaCO}_{3} \text { were studied } \\
\text { - Results showed limited changes with the addition of elastomer and } \mathrm{CaCO}_{3} \text { on the mechanical } \\
\text { properties of the recycled PP }\end{array}$ \\
\hline
\end{tabular}




\section{Material and methods}

After conduct a detailed functional analysis where is to analyze the needs expressed by the company and to characterize, identify and prioritize the features of the product during its lifecycle in order to develop the functional specifications to facilitate and clarify the vision in the choice of the optimal solutions. This method is divided into several points:

- State and validate the needs to be met.

- Identify the elements of the environment of operation of the product.

- Establish the diagram of Octopus.

- Identify the functions of service.

- Characterize the service functions identified.

- Prioritize the services functions.

- Develop the specifications works l.

- Choice the solutions

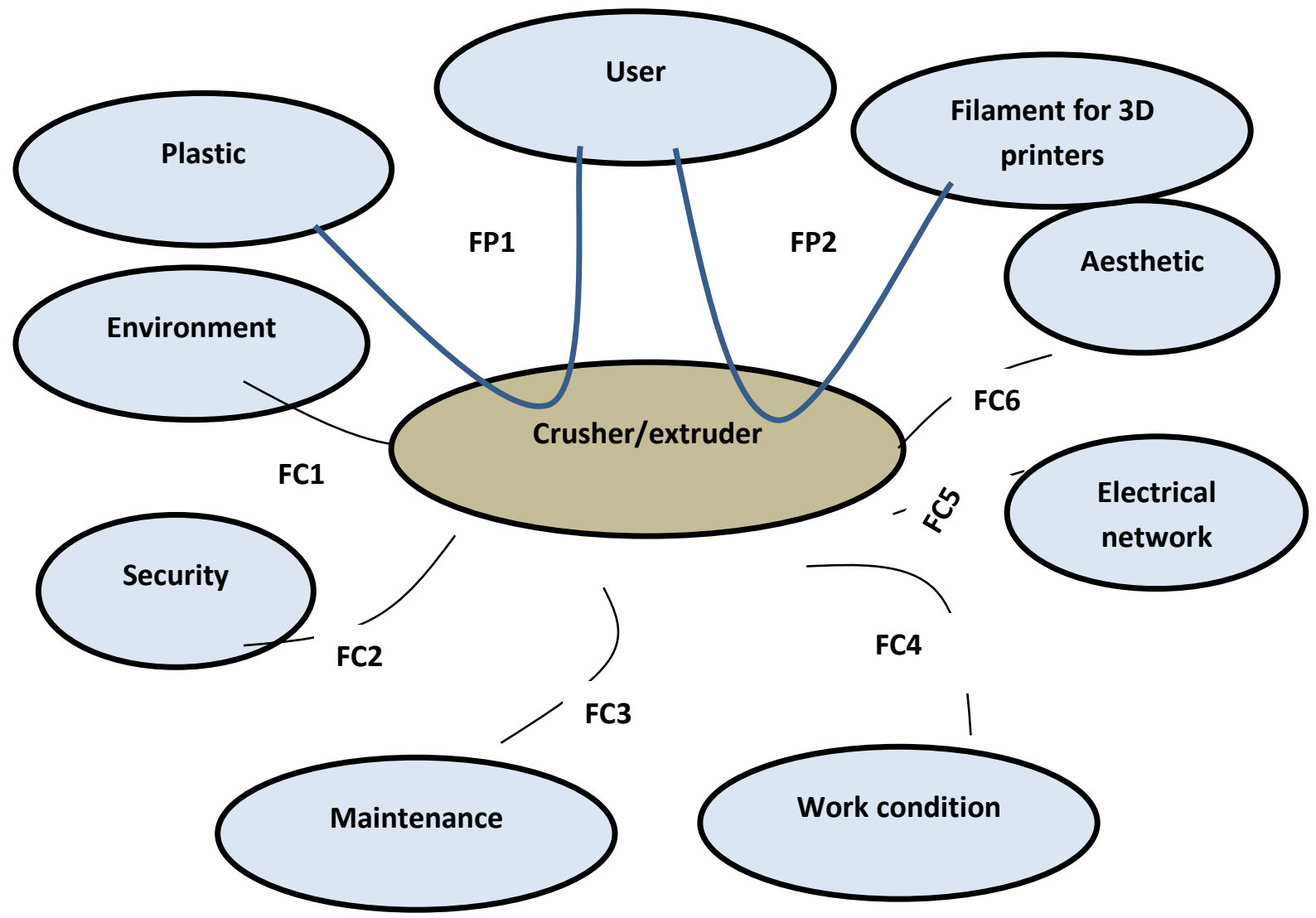

Figure 1. functions Identification of service functions: Octopus flowchart

In order to prioritize the famous features of services, we adopt the principle of cross sort by comparing the functions one by one using a matrix with the award notes as indicated by suite:

The goal is to determine an optimal solution which meets functional requirements. For this, we have adopted a methodology structured taking into account the elements and requirements of the 
specifications to achieve a result that meets the needs. After conducting the study of plastic and existing crusher and extruder, it found that most plastics have good thermal properties, chemical and physical. However, consulting a few suppliers of three-dimensional printers we find that most of these revolutionary printers use filaments PLA and ABS as raw material in the first place. Secondly, they also use PE-HD. For this, working on these 3 types of plastics and set our choice as to the solutions that we adopt in this project and after studying the existing Circular blades mill, is a bit more complicated in its machining (gears and several levels...) but it's possible with our means. In addition, it works with two engines (and in the case of 4 or 6 shaft) and it contains no hopper and for the extruder, usually the extruders are all the same but they defer one compared to the other by a few technical details such as dimensions, mechanical solutions, heating method, type of screw extrusion. (Oussai \& Zoltan, 2017).

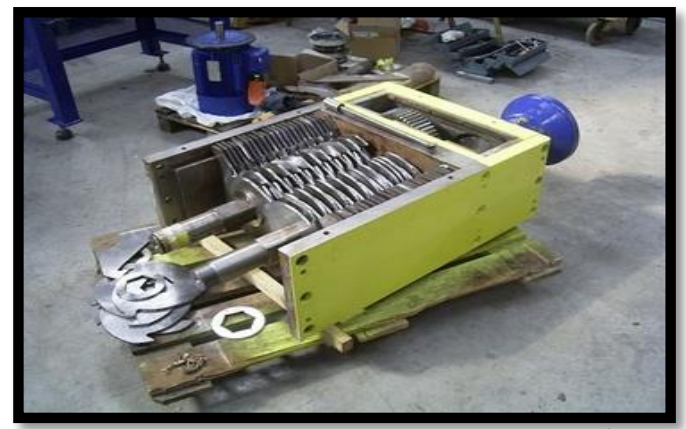

Figure 2. setting the choice for crusher ${ }^{3}$

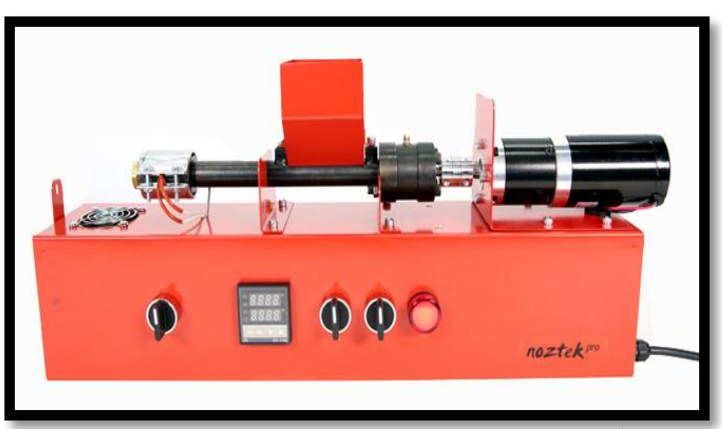

Figure 3. setting the choice extruder

Functional analysis is to analyze the needs expressed by the company and to characterize, identify and prioritize the features of the product during its lifecycle in order to develop the functional specifications to facilitate and clarify the vision in the choice of the optimal solutions This part enabled us to choose an optimal solution for various components of this project

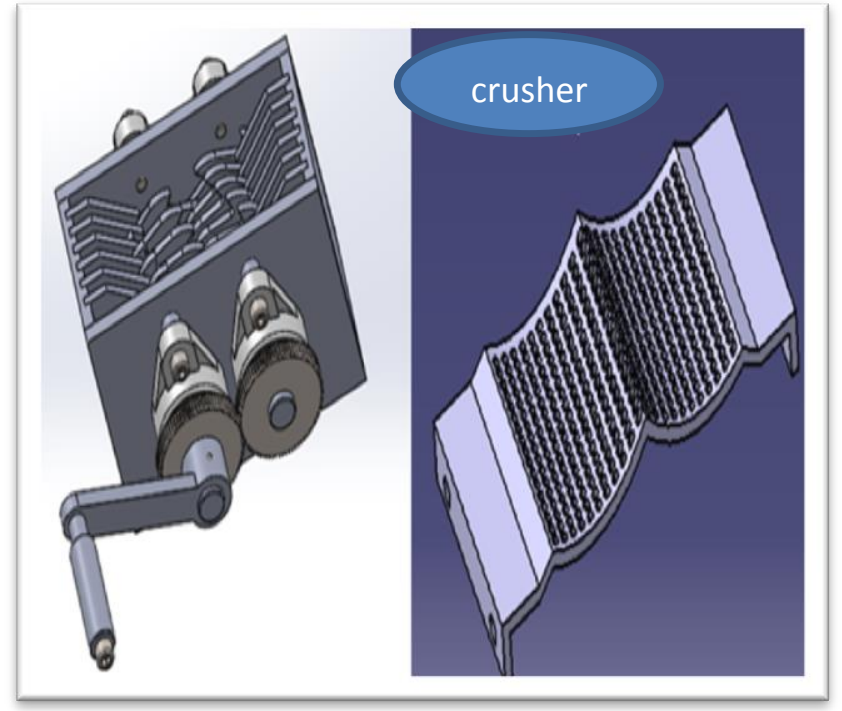

Figure 4. Presentation of solutions for crusher $^{3}$

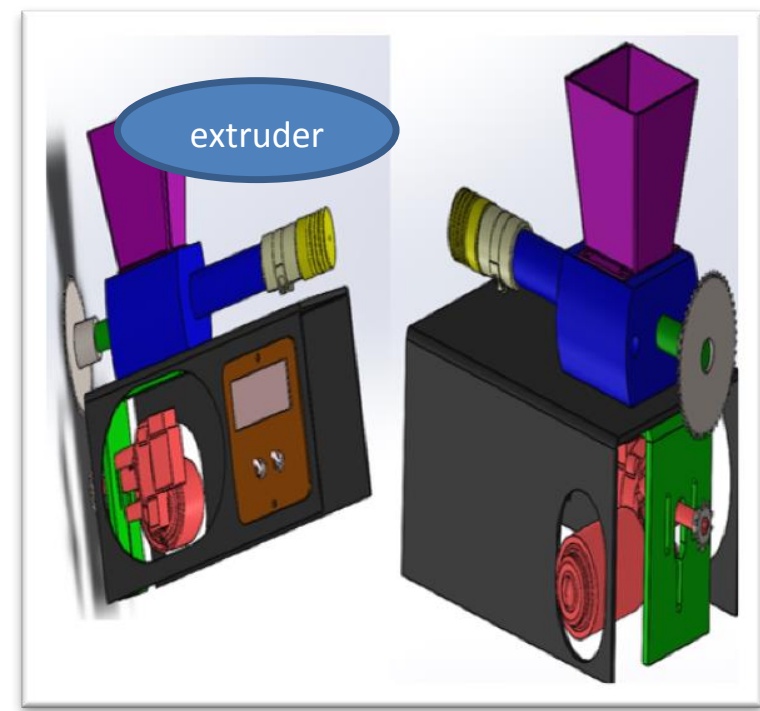

Figure 5. Presentation of solutions extruder ${ }^{3}$

For the design and sizing the crusher, the process will begin with description system, as well as the scheme of kinematics. Then we will detail the design of the various blocks of the system and we will assemble; specifying the technical details while using the CAD SOLIDWORKS 2015 software. After this, we will conduct calculations and dimensioning of the Crusher 


\section{Results and discussions}

Recent research points the way towards chemical recycling methods with lower energy requirements, compatibilization of mixed plastic wastes to avoid the need for sorting, and expanding recycling technologies to traditionally non-recyclable polymers.

We mentioned the recycling technologies from which we concluded that mechanical recycling is the only widely adopted technology for large-scale treatment of plastic solid waste. The main steps were the removal of organic residue through washing, followed by shredding, melting, and remoulding of the polymer, which is often blended with virgin plastic of the same type to produce a material with suitable properties for manufacturing.

There are limitations to mechanical recycling technologies because each type of plastic responds differently to the process depending on its chemical makeup, mechanical behaviour, and thermal properties. Temperature-sensitive plastics, composites, and plastics that do not flow at elevated temperatures (as in the case of thermosets) cannot be processed mechanically. Consequently, only two types of plastic are recovered and recycled with mechanical processes: poly (ethylene terephthalate) (PET) and polyethylene, which represent 9 and 37\% of the annual plastic produced, respectively. All other plastic solid waste is either not recovered or in amounts representing less than $1 \%$ of production. According to the most recent U.S. Environmental Protection Agency report, a mere 8.8\% of all plastic produced in the U.S. annually is recovered from municipal solid waste and then incinerated, recycled, or industrially composted. The recycling rate is slightly higher in Europe, at $\sim 30 \%$ for plastic waste. 4

In my previous research supervised by Dr Zoltán Bártfai, I found solution for grinding and extruding plastic using machines with two different parts: crusher, extruder and mixed it together.

The crusher consisted of 12 blades twelve anvil-blades, right axis, left axis, cover, sieve, fourteen casing spacers, four nuts, two pins and crank. 3 ,the extruder consists of: hopper, room of extrusion, heating collar, chain transmission, nozzle, engine support, engine, control panel and the extruder support. The component which is most important in the extruder is the extrusion screw that acts like a screw pump, fits in the room.

As, we have stated previously, the function of the screw is to move the pieces of plastic inserted through the hopper along the length of the room.

As long as the plastic material progress horizontally towards the bottom of the room, the heating element melted, and so the worm screw also works to mix the plastic during the melting. The screw is important to ensure the homogeneity of the filament, which is one of our system requirements.

In order, to achieve the functions requested by the screws we designed it with three geometrically different parts.

- Recharge area: the depth in this area is constant.

- Compression area: the depth gradually decreases and the matter begins to melt.

- Pumping area: the depth is again constant and lower than in the recharge area. 


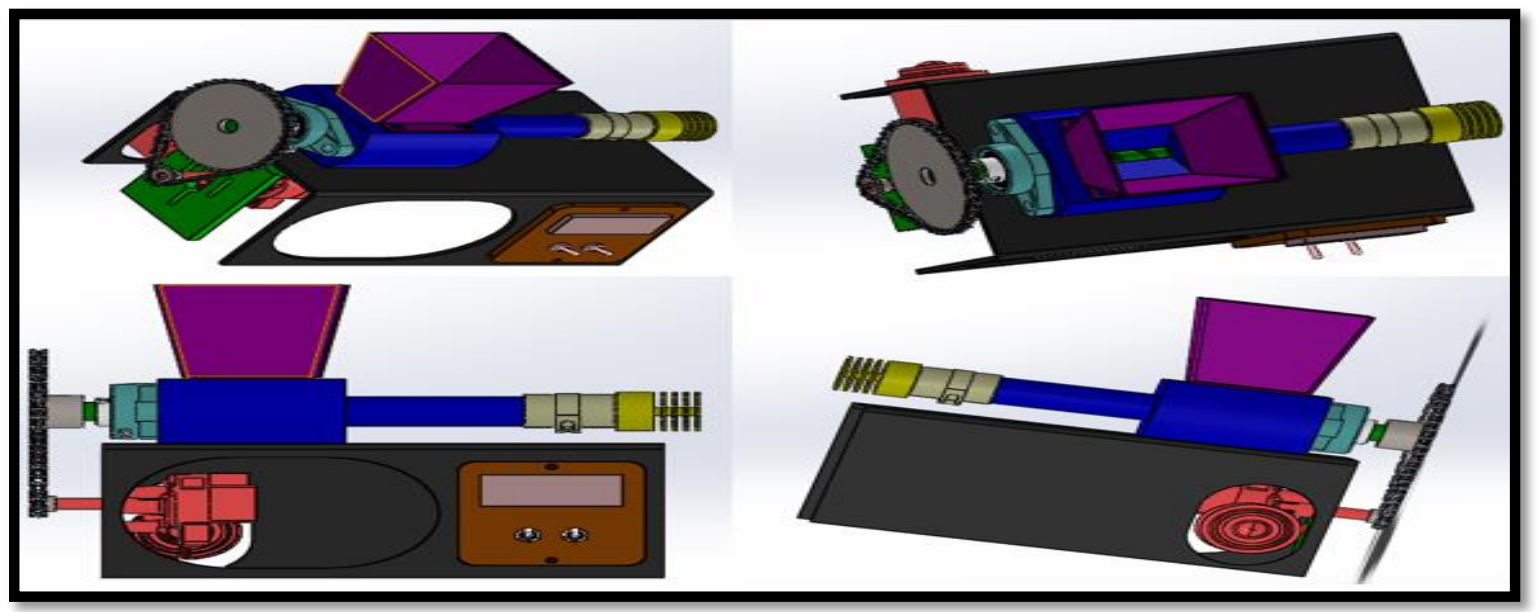

Figure 6. overview of the design of extruder ${ }^{3}$

Finally, after theoretical calculation and sizing which we size the screw extruder and choosing the the engine for that and then studying he resistance and validation of the structure we conclude that the design proposed during this calculation responded to the requirements of the specifications functional especially from the point of view of the system.

\section{Conclusion}

This paper study the types of plastics and diagnose the pollution caused by the latter. This project also allowed to design and size a recycling station of plastic into filaments for three-dimensional printers , this station which will contribute to the fight against pollution.

The station consists of two machines for grinding of the plastic and the other for the extrusion of the desired filaments. We were able to make a theoretical academic study on both machines and also, we designed with SOLIDWORKS 2015. The theoretical study is spread of the mechanical calculations necessary to the design and validation of the structure using the tools. As the prospect of this project, we want to complete the achievement of this station while completing the crusher and extruder mechanically. Then switch to electric and electronic parts (introduction of engines, sensors and wiring...). Next, we want to automate this station to make it autonomous.

In the end of this paper, we show the environmental consequences of plastic solid waste are visible in the ever-increasing levels of global plastic pollution both on land and in the oceans. But although there are important economic and environmental incentives for plastics recycling, end-of-life treatment options for plastic solid waste are in practice quite limited.

Pre-sorting of plastics before recycling is costly and time-intensive, recycling requires large amounts of energy and often leads to low-quality polymers, and current technologies cannot be applied to many polymeric materials. ${ }^{5}$

Recent researches point the way toward chemical recycling methods with lower energy requirements, compatibilization of mixed plastic wastes to avoid the need for sorting and expanding recycling technologies to traditionally nonrecyclable polymers. 
Mechanical recycling is the only widely adopted technology for large-scale treatment of plastic solid waste. In it the main steps are the removal of organic residue through washing, followed by shredding, melting, and remoulding of the polymer, which is often blended with virgin plastic of the same type to produce a material with suitable properties for manufacturing. ${ }^{6}$

\section{Reference}

[1] Al-Salem, S. M., Lettieri, P., \& Baeyens, J. (2009) Recycling and recovery routes of plastic solid waste (PSW): A review. Waste Management. 29(10) pp. 2625-2643. https://doi.org/10.1016/J.WASMAN.2009.06.004

[2] Flores, M. C. (2008) Plastic Materials and Environmental Externalities Plastic Materials and Environmental Externalities: Structural Causes and Corrective Policy Table of Contents Plastic Materials and Environmental Externalities Plastics Recycling: Law and Economics. Lethbridge Undergraduate Research Journal. 3 pp. 2-7.

[3] Oussai, A., \& Zoltan, B. (2017) Design and Sizing of a Plastic Recycling Station Into Filaments for 3D Printer. In Engineering, Agriculture and Green Industry Innovation (pp. 1-6). Budapest: Synergy International Conferences.

[4] Frigione, M. (2010) Recycling of PET bottles as fine aggregate in concrete. Waste Management. 30 (6) pp. 1101-1106. https://doi.org/10.1016/J.WASMAN.2010.01.030

[5] Saikia, N., \& de Brito, J. (2014) Mechanical properties and abrasion behaviour of concrete containing shredded PET bottle waste as a partial substitution of natural aggregate. Construction and Building Materials. 52 pp. 236-244. https://doi.org/10.1016/J.CONBUILDMAT.2013.11.049

[6] Sonawane, Y. N., \& Chitte, C. J. (2017) Waste Coconut Shell as a Partial Replacement of Coarse Aggregate in Concrete Mix - An Experimental Study. International Journal of Science and Research. 5(4) pp. 2014-2016. 